D. Kukla ${ }^{1}$, A. Bałkowiec ${ }^{2}$, P. Grzywna ${ }^{1}$

${ }^{1}$ Institut of Fundamental Technological Research Polish Academy of Science, 02-106 Warszawa, ul. Pawińskiego 5b,dkukla@ippt.gov.pl

${ }^{2}$ Warsaw University of Technology, Faculty of Materials Science and Engineering, 02-507 Warsaw,Woloska str.141,Poland,abalkowiec@inmat.pw.edu.pl

\title{
EVALUATION OF MICROSTRUCTURAL CHANGES OF S235 STEEL AFTER ROLLING ON THE BASIS OF MICROSCOPIC OBSERVATIONS AND EDDY CURRENT NON-DESTRUCTIVE METHOD
}

\begin{abstract}
The aim of the study was to determine the microstructure and residual stress changes which appears in the S235 steel (which is designed for operating in elevated temperatures) during cold rolling. The changes of orientation, both morphological and crystallographic, are occurring as a result of the rolling process. Analysis of the results allowed to determine the change in grain morphology (size, shape), determination of grain morphology of the rolling direction and determination of crystallographic texture. The dislocation density present in the material before and after the cold rolling process was estimated on the basis of dislocation structure images obtained via transmission electron microscope. The observed microstructural changes were correlated with the results of nondestructive testing using eddy current method. They allowed for the identification of the state of stress measured on three different surfaces of the rolled sheet (parallel and perpendicular to the rolling direction). As the result the usability of using the non - destructuve techniques of stress level determination was proved.
\end{abstract}

Key words: S235 steel, microstrcture, eddy current, cold rolling

\section{INTRODUCTION}

Determining the microstructure changes which appeares in the investigated steel as the result of carried cold rolling process is important for estimation of residual stress, which has strong influence on mechanical properties of the deformed material. The goal of the presented experiment was to specify microstructure changes, formation of crystallographic texture and residual stress increasement in the S235 sheet.

Analysis of literaure data gives a general knowledge about the qualitative type of the microstructure changes during cold rolling, however the quantitative description of these changes is specific for the defined material and strongly depends from the parameters of the rolling procces. These facts encourages to run the experiments for each of the specific materials and process.

Presented investigations includes a number of research techniques, i.e. EBSD and TEM. Stereological parameters were determined for the initial material and for the material after cold rolling procces. Comparison of the dislocation density for the initial sample and for the 
material after deformation was conducted. These investigation were conducted on the samples which needed special preparation before the measurements.

As in the industrial practice destructive investigation are not always possible to conduct, the non-destructive testing were also carried out. Eddy current investigation was made on three different surfaces (rolling surface of the plate and cross-sectional areas on the longitudinal direction of rolling). The analysis of eddy current characteristic changes using "lift-off" effect on the rolled sample surface was done. Obtained results proved possibility of identification areas which have higher level of residual stress.

\section{METHODOLOGY}

The investigations were conducted on the steel of S235 type with different deformation level. Initial sample (S) was a $12 \mathrm{~mm}$ thick, hot rolled sheet. The initial material was cold rolled to 8 and $6 \mathrm{~mm}$ sheet and noted as S1W $(8 \mathrm{~mm})$ and S2W $(6 \mathrm{~mm})$ respectively.

Samples, which were observed with the use of light microscopy, were prepared with the standard metallographic techniques (mechanical polishing) and etched with $10 \%$ Nital. Samples fot the EBSD investigtions were mounted in the conductive resin and mechanically polished with the final step polishing with the use of acidid $\mathrm{SiO}_{2}$ suspension. Samples for TEM observations were prepared with the use of electrochemical thining. Non-destructive evaluation was done with the use of eddy current method on initial sample and S2W (6 mm thick).

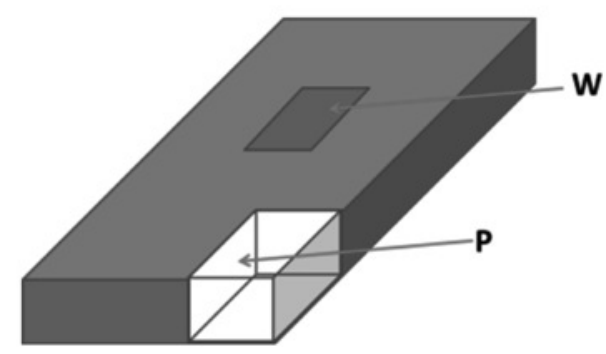

Fig. 1. Sketch of investigated surfaces, $W$ - surface parallel to the surface of the sheet, $P$ - cross section perpendicular to the surface of sheet and parallel to the rolling direction

Stereological analysis of the microstructure was done on the LM (light microscopy) images. Analyzed surace was the $\mathrm{W}$ cross section, $0.2 \mathrm{~mm}$ below the sample surface. The equivalent diameter $\left(\mathrm{d}_{2}\right)$ of the observed grains, normal distribution of the $\mathrm{d}_{2}$ and the rose graph of the grain elongation in dependace from the rolling direction was provided. Additional metallographic observation on P cross - section for samples S1W and S2W were done.

Determination of the crystallographic texture for samples S, S1W, S2W was done with the use of EBSD technique. The investigations were done with the use of Hitachi SU70 analytical microscope with EBSD detector. The acceleration voltage was $20 \mathrm{keV}$, sample tilt was 70 degree. Obtained orientation maps enabled analysis of crystallographic orientation of the analyzed area.

TEM observations were carried on JEOL JEM 1200 EX. Microstructure images were used for estimation of density dislocation on samples S, S1W and S2W. On each image 5 straight lines with random orientation were applied. The intersection of dislocation and lines were 
counted [1]. Analyzed areas were obsereved with the magnification of 50000 and 100000 . Dislocation density was estimated from the equitation:

$$
\rho=\mathrm{N} / \mathrm{Lrt}
$$

where,

$$
\begin{aligned}
& \mathrm{N} \text { - number of dislocation - Line intersection } \\
& \mathrm{Lr}-\text { the total length of all lines } \\
& \mathrm{T}-\text { sample thickness (established } \mathrm{T}=200 \mathrm{~nm} \text { ) }
\end{aligned}
$$

The last type of research carried out in the framework of the presented study was non destructive and was based on the indicated eddy current signal analysis of the different surfaces of samples S235 after rolling. In this case, the phase angle was measured by the linear effect of distance between the tested surface of the steel sheet and probe. This parameter is dependent on many factors, such as: internal stress changes, local changes in the composition, density or the phase composition [2-7], and therefore an attempt was made to use it to identify changes in the rolling surface. The measurement of the phase angle was made using the software available in the commercial Zetec device. Curve slope angle was measured for the rolled surface and the two other surfaces of the samples with thickness of 6 (S1W) and 8 (S2W) mm. For each sample's surface a measurement of the lift-off curve slope according the scheme showed on the figure 2 .

This procedure allowed to identify local changes in electrical conductivity and the magnetic permeability caused by deformation.

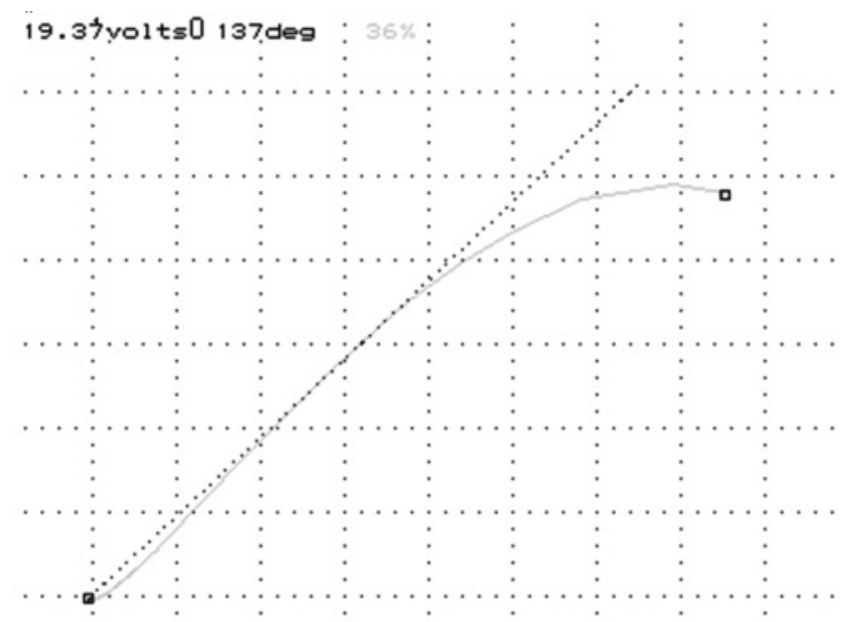

Table 1. Measurement parameters

\begin{tabular}{|l|c|}
\hline Frequency & $\begin{array}{c}\text { Estimated depth of } \\
\text { penetration [mm] }\end{array}$ \\
\hline $100 \mathrm{kHz}$ & 0.635 \\
\hline $750 \mathrm{kHz}$ & 0.4572 \\
\hline $1 \mathrm{MHz}$ & 0.3048 \\
\hline
\end{tabular}

Fig. 2. Measurement technique of the phase angle signal of eddy current in dependance from distance (lift-off)

Measurements were made for three excitation current frequency: $100 \mathrm{kHz}, 750 \mathrm{kHz}$ and 1.5 MHz, which allowed for evaluation of changes in stress orientations determined by changes in the function of distance from the sample surface. Table 1 shows an estimate of the depth of penetration of the applied current frequency. 


\section{RESULTS}

\section{Microstructure observations}

Observations of metallographic samples revealed the ferritic-perlitic structure with wide range of grain size (see figure 3). Grain size refinement is observed for sample S2W. In none of the observed W cross - section (sample S, S1W and S2W) the morphological texture was observed.

Images of microstructure observed on $\mathrm{P}$ cross - section (parallel to rolling direction and perpendicular to sheet surface) are shown on image 4. Figure 4a shows the microstructure of sample S1W. Parallel to the sample surface, the area with clearly visible grain size reduction is visible. Figure $4 \mathrm{~b}$ show the microstructure of sample $\mathrm{S} 2 \mathrm{~W}$. The size reduction of grain dimensions is clearly visible as well as grain shape banding.
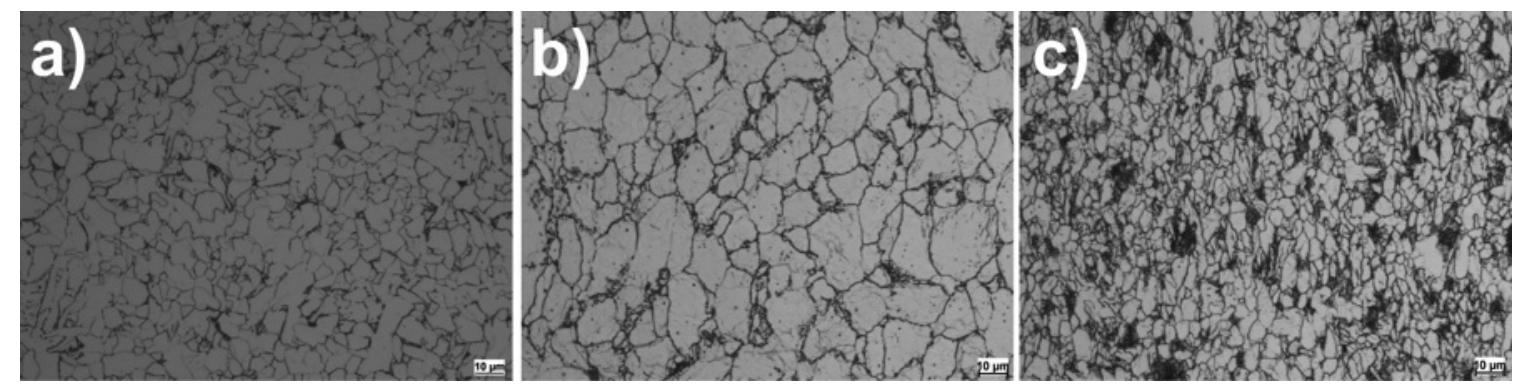

Fig. 3. Microstructure of $S 235$ steel of the initial sample $S(A)$ and after cold rolling: sample $S 1 W(B)$ and $S 2 W$ (C), W cross - section
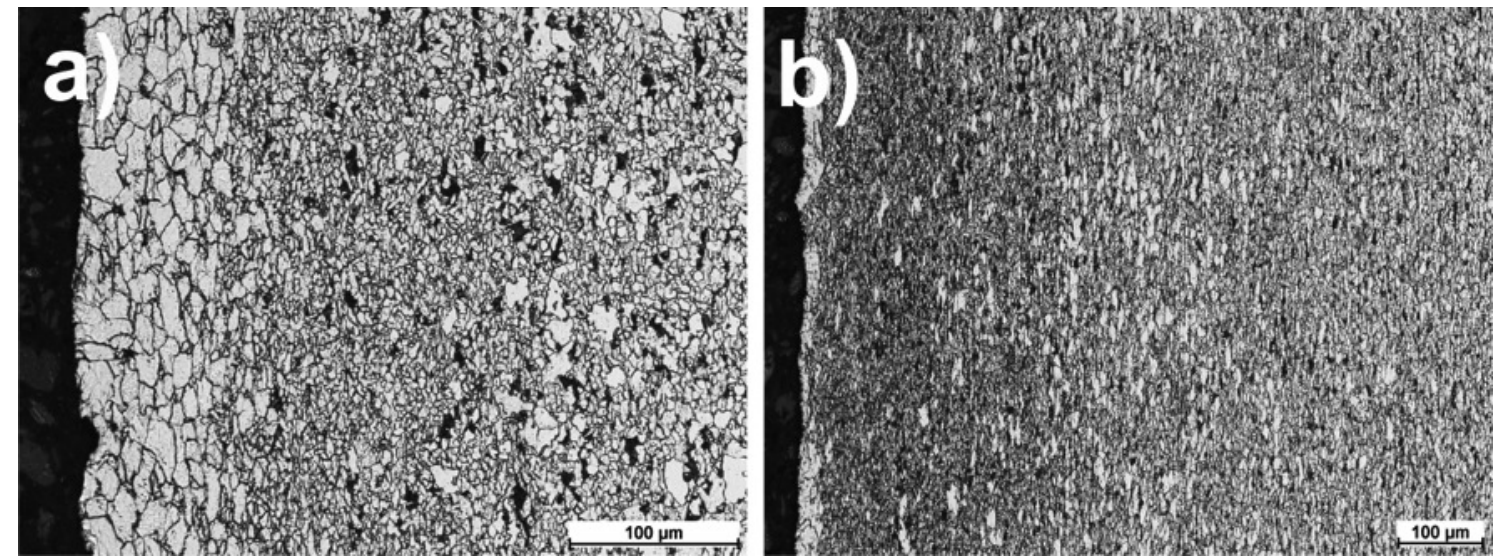

Fig. 4. Microstructure of S235 steel after cold rolling: sample S1W (B) and S2W (C), P cross - section

Results of stereological analysis are shown in table 2 and figure 5. The average equivalent diameter is, as expected, distinctly smaller for the S2W sample in comparison to sample S. Unexpected result was received for sample S1W for which the $d_{2}$ is much higher than the $d_{2}$ obtained for sample S. To fully understand the observed phenomena, the normal distribution of $\mathrm{d}_{2}$ value were prepared (see figure 5). 
Table. 2. Average equivalent diameter (d2) $[\mu m]$ in sample S, S1W and S2W

\begin{tabular}{|l|l|l|l|}
\hline & Sample S & Sample S1W & Sample S2W \\
\hline $\mathrm{d}_{2}(\mathrm{Cv})$ & $5.87(3.62)$ & $7.95(5.30)$ & $2.99(1.50)$ \\
\hline
\end{tabular}

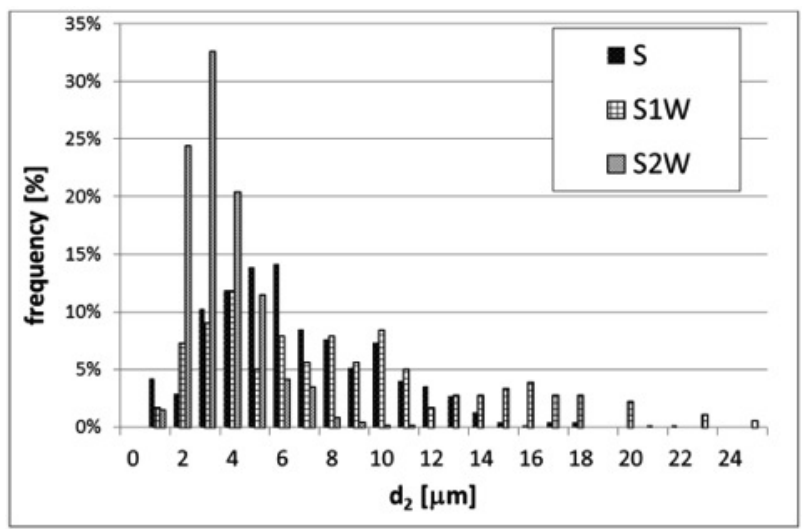

Fig. 5. Normal distribution of the eqvivalent diameter $\left(d_{2}\right)$ for sample $S, S 1 W$ and $S 2 W$

Analysis of the normal distribution of equivalent diameter enhances the opportunity to analyze the microstructure changes and allow to fully understand the unexpected result for the mean value of $\mathrm{d}_{2}$ in sample $\mathrm{S} 1 \mathrm{~W}$.

Normal distribution of $\mathrm{d}_{2}$ for sample $\mathrm{S}$ is close to theoretical shape of Gaussian curve. The maximum frequency appears for the grain size between 5 and 6 um. For the sample S1W the maximum value of frequency appears for the value between 4 and $5 \mu \mathrm{m}$. The shape of the S1W normal distribution exhibits wide plateau for the grain size from the range between 3 and $10 \mathrm{um}$, which explains the high mean value of $\mathrm{d}_{2}$ for sample S1W. For the sample $\mathrm{S} 2 \mathrm{~W}$ the shift in the direction of lower $\mathrm{d}_{2}$ values is observed. The highest frequency of appearance is observed for grain size 3-4 $\mu \mathrm{m}$. The shape of normal distribution is close, like for the sample $\mathrm{S}$, to Gaussian curve.

Analysis of shape of grains and its elongation in dependence from rolling direction was done with the use of rose graph. The diagrams show the angle between the longest chord of the analyzed grain and the rolling direction. Obtained results are shown in Fig. 6.

A)

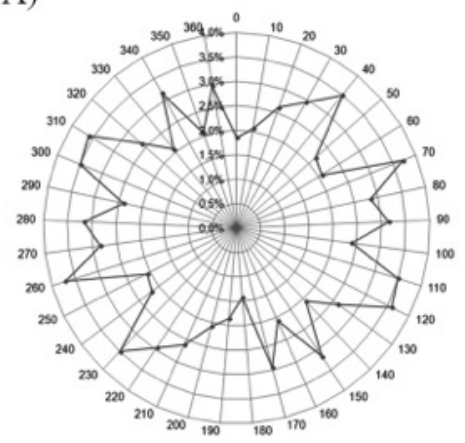

B)

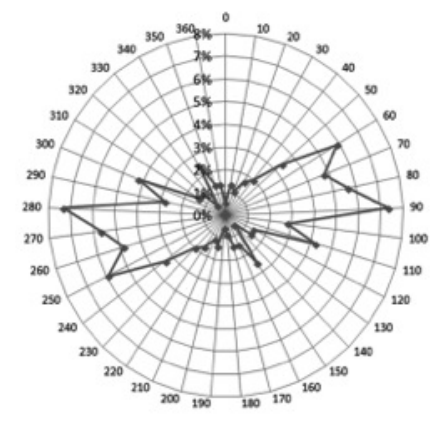

C)

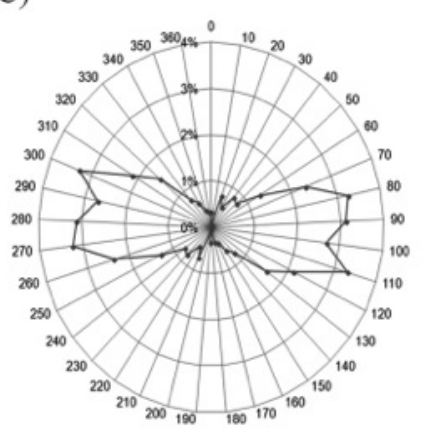

Fig. 6. Rose graph of angle between the longest chord of the grain and rolling direction for sample S (A), S1W (B) and sample S2W (C) 
Rose graph obtained for sample S (initial sample) do not show any dominant angle of deviation, which confirms that in the initial materials no morphological texture is observed. For the samples subjected to cold rolling process the morphological texture, parallel to the rolling direction.

EBSD measurements were conducted on the surface paralell to the sample surface and after removing of $0.2 \mathrm{~mm}$ of material thickness (W cross - section). The orientation maps were prepared. The results are shown on figures 7 and 8 . The map of the sample S2W shows the structure rich in low angle grain boundaries which is common for the deformed material.

The measurement of crystallographic texture via EBSD were made for two samples: S and S2W and the W cross - section. Obtained pole figures $\{111\}$ were prepared for the EBSD orientation maps, which were shown of figure $7 \mathrm{a}$ and $7 \mathrm{c}$. For the $\mathrm{S}$ sample no texture components can be recognized. The crystallite orientation is chaotic which proves that no crystallographic texture can be defined for S sample. Pole figures of sample S1W and S2W are typical for the cold rolled materials. For the S1W sample the component typical brass $\{1$

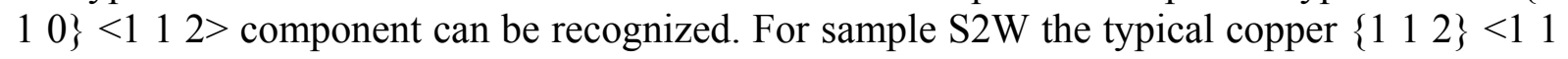
$1>$ component is visible.
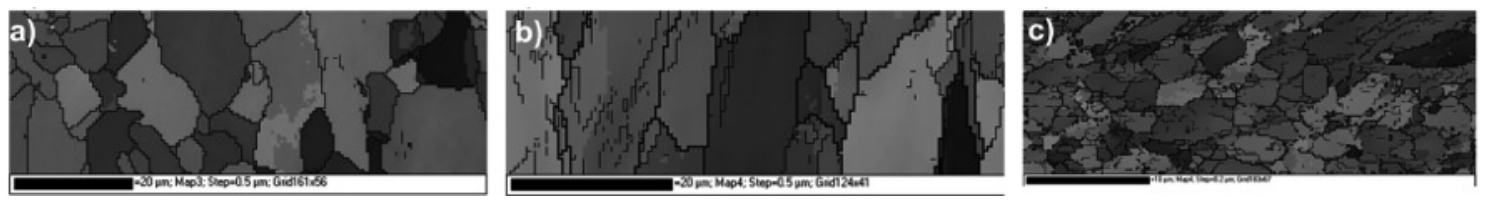

Fig. 7. Orientation map for sample S (A), S1W (B) and S2W (C)
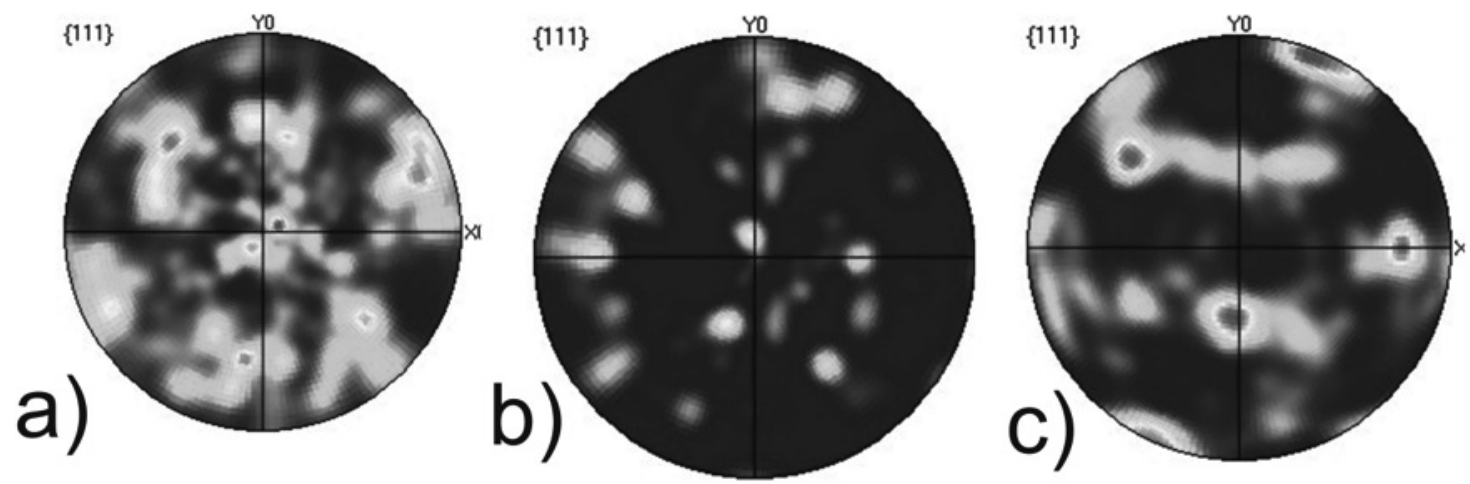

Fig. 8. Pole figure for sample $S(A), S 1 W(B)$ and $S 2 W(C)$

Calculating the dislocation density was conducted on images taken at the magnifications of 50,000 and 100,000. Examplary images of the dislocation structures and measurement lines are shown on figure 9 for samples S and for sample S2W on figure 10. The number of estimated dislocation for analyzed samples are shown in table 3. 

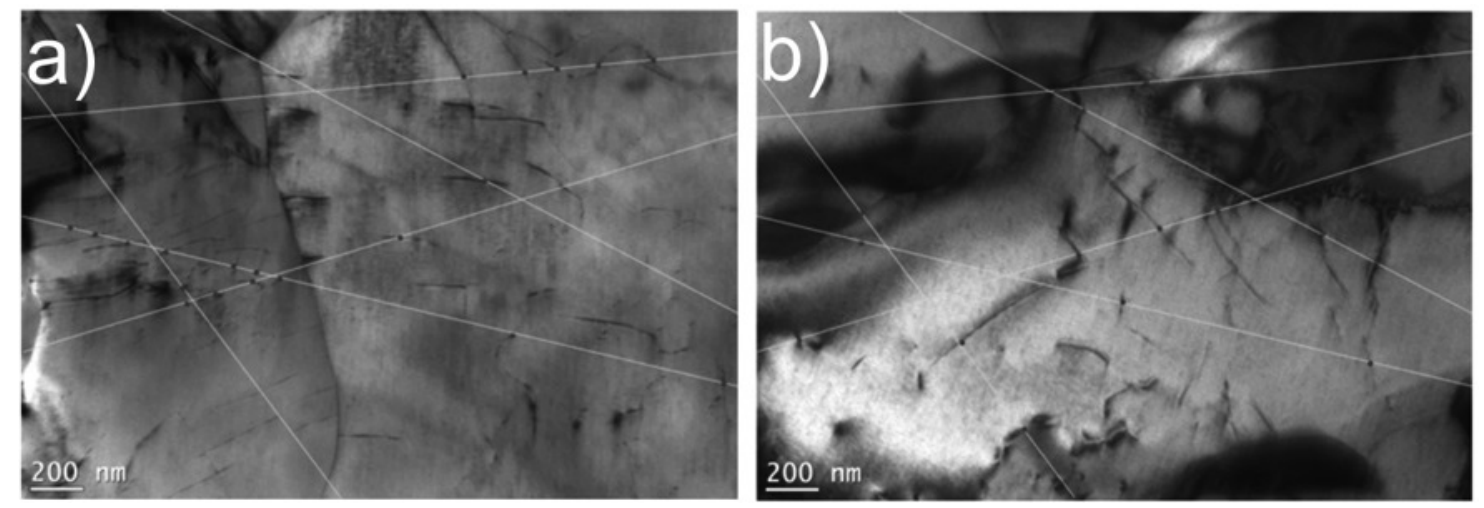

Fig. 9. Dislocation structure of S235 (sample S) with visible dislocation and measurement lines, the number of intersection is 21 for image $A$ and 17 for image $B$
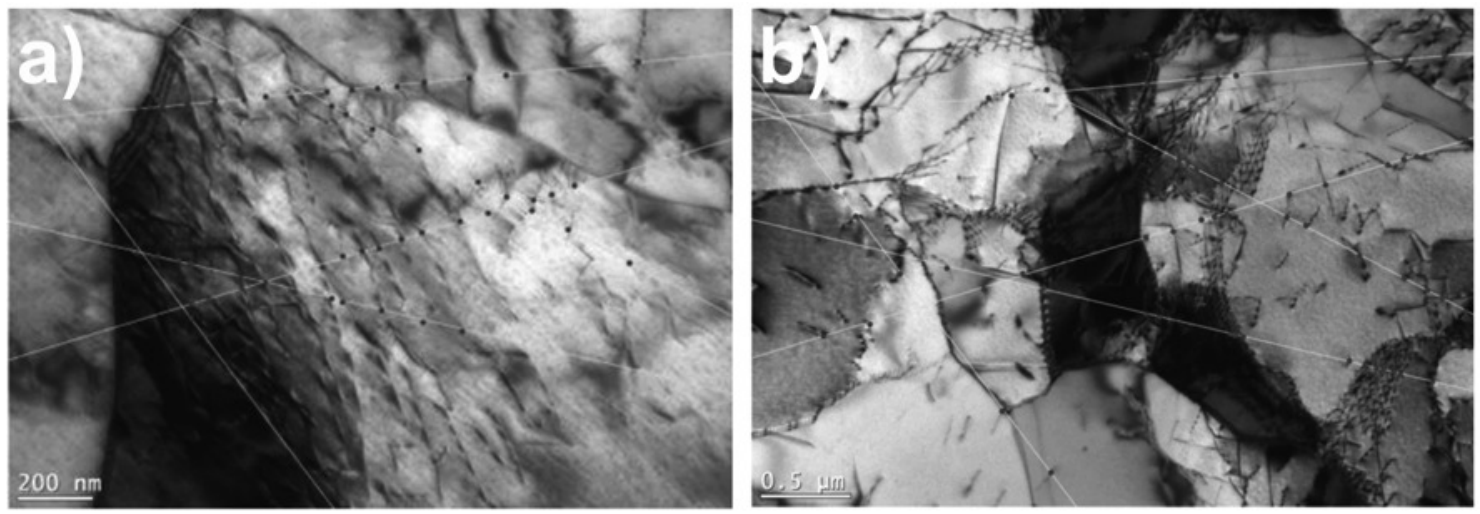

Fig. 10. Dislocation structure of S235 after cold rolling (sample S2W) with visible dislocation and measurement lines, the number of intersection is 48 for image $A$ and 51 for image $B$

Table.3. Mean value for dislocation density in sample S and S2W

\begin{tabular}{|l|c|}
\hline Sample & Dislocation density \\
\hline S (initial state, $12 \mathrm{~mm}$ thick) & $5,5 \times 10^{+12} \mathrm{~m}^{-2}$ \\
\hline S1W (cold rolled, $8 \mathrm{~mm}$ thick) & $9,14 \times 10^{+12} \mathrm{~m}^{-2}$ \\
\hline S2W (cold rolled, $6 \mathrm{~mm}$ thick) & $1,1 \times 10^{+13} \mathrm{~m}^{-2}$ \\
\hline
\end{tabular}

Results clearly show that the increase of dislocation number for samples after cold rolling in comaprison to initial state, however the higher number of observed dislocation was expected. This fact can be explained by low dimension of analyzed area (TEM sample) which can make the analyzed volume of the material insufficient for proper estimation of dislocation density. Other explanation is possible anihilation of dislocation being a result of residual stress relaxation during TEM samples preparation.

Measurements of the phase angle of the eddy current signal were made on three mutually perpendicular surfaces of the steel sheet showed dispersion of the values of this parameter. Observed differences can be caused by changes in the structure of the material mainly understand as the residual stresses in the materials surface layer. The results of these tests carried out on the rolled sheet of 6 and $8 \mathrm{~mm}$ thickness are shown in Figure 11. 
a)

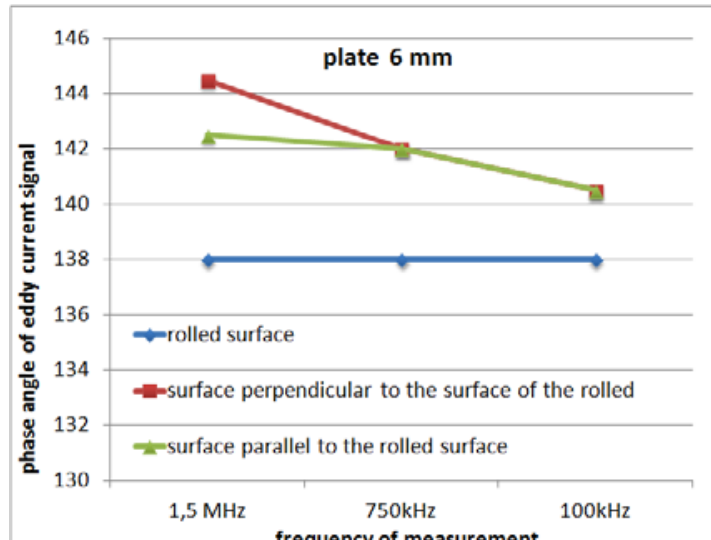

Fig. 11. Changes in the phase angle as a function of frequency for the three surfaces of the sheet with a thickness of $6 \mathrm{~mm}(\mathrm{a})$ and $12 \mathrm{~mm}(\mathrm{~b})$

Based on the presented results it can be concluded that the value of the measured parameter obtained on the rolled surface, which according to mechanical treatment, is defined with the highest stress level, has the lowest and constant angle values, which is not dependent of the sheet thickness or the measurement frequency.

Different level of the phase angle was obtained for the two measured surfaces, perpendicular to the rolled surface, however the surface which was parralel to the rolling direction is defined with the middle value of the phase angle, which can be correlated with the stress pattern. This results proves that the eddy current non destructive method can be used for identification of the surface stresses pattern changes in coventional range.It should be noted that phenomena of disrepancy of obtained values of phase angle obtained for each invetigated surface, is the most distinct for measurement frequency of $1.5 \mathrm{MHz}$, when the penetration depth is $0.3 \mathrm{~mm}$.Observed values are weakaned for the frequency of $700 \mathrm{kHz}$, which corresponds to penetartion depth of $0.6 \mathrm{~mm}$.Observed relationship can be results of cumulated stresses cused by cold rolling process. This result is presented on figure 12 .

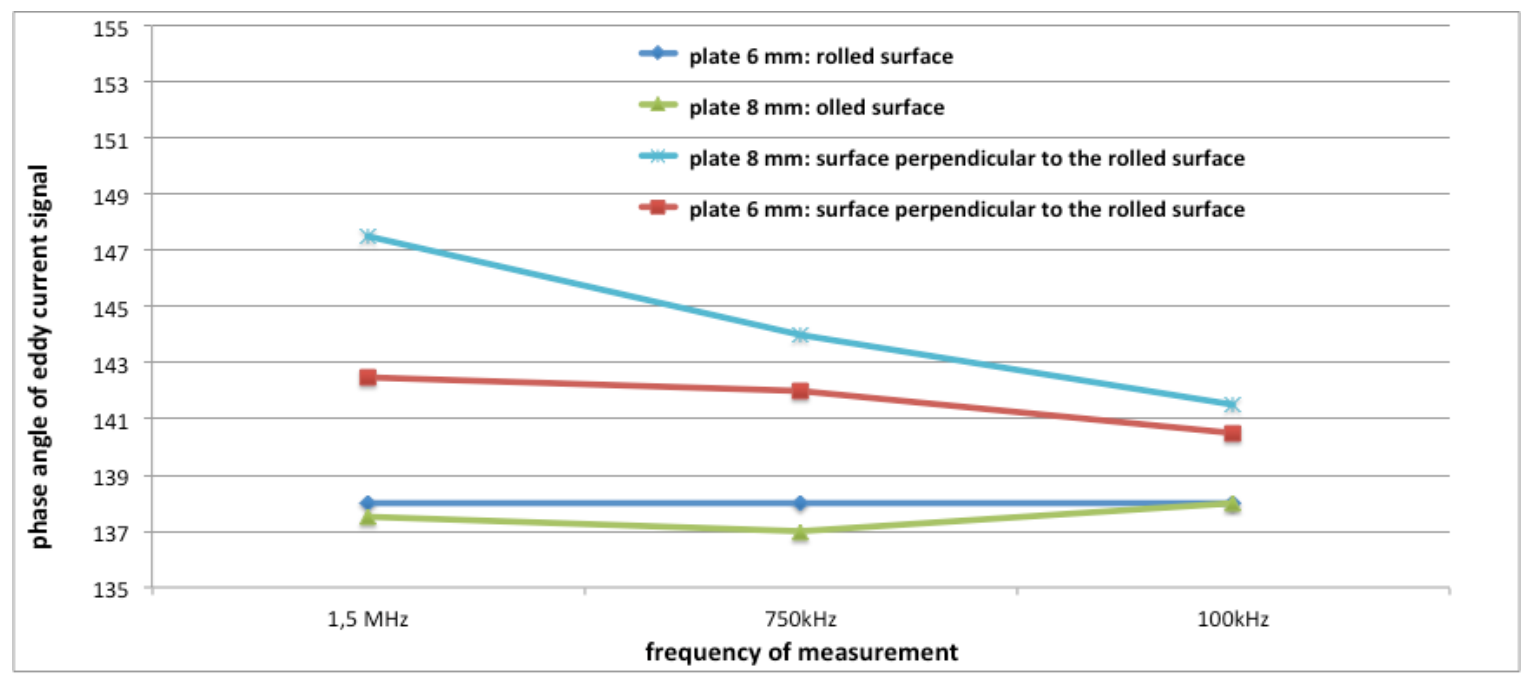

Fig. 12. Changes of the phase angle value as a function of frequency for sheet with a thickness of 6 and $8 \mathrm{~mm}$ 


\section{CONCLUSION}

The presented research allowed for preparation of qualitative characteristic of microstructural changes in the S235 steel sheets during cold rolling. These changes encloses changes in the grain shape, size and elongation, crystallographic and morphological texture as well as dislocation density and surface residual stresses. A trial of correlation of qualitative results of microstructure changes and the results of non - destructive technique investigations, was made. It has been proved that, with the specific data, defined on reference samples, enables estimation of residual stress level on materials after deformation processes, without the need of sampling.

Preliminary investigation with the use of non-destructive method of eddy current measurements has shown correlation of the results which encourage for further investigations. The elaborated methodology of non - destructive estimation of sample residual stresses value, can be applied i.e. for the evaluation of quality of conducted surface mechanical treatment and for localization of microstructural notch, which are responsible for stresses concentrator.

\section{ACKNOLWEDGEMENT}

The study was conducted as part of the PBS project "Development of magnetic methods to assess the state of stress in engineering materials in particular anisotropic" No. 179032 funded by NCBiR

\section{REFERENCES}

1. Norfleet D.M., Dimiduk D.M., Polasik S.J., Uchic M.D., Mills M.J.: Dislocation structures and their relationship to strength in deformed nickel microcrystals, Acta Materialia, 56 (2008), 29883001 .

2. Kukla D., Grzywna P., Zagórski A.: Development evaluation fatigue degradation of P91 steel based on changes in the phase angle of theeddy current signal [in Polish], Welding Technology Review, 13 (2012), 8-11.

3. Oka M., Tsuchida Y., Yakushiji T., Enokizono M.: Fatigue Evaluation for a Ferritic Stainless Steel (SUS430) by the Eddy Current Method Using the Pancake-Type Coil. Idee Transactions On Magnetic 46-2 (2010).

4. Kukla D., Kowalewski Z., Grzywna P., Kubiak K., Assessment of fatigue damage development in power engineering steel by local strain analysis, KOVOVE MATERIALY-METALLIC MATERIALS (ISSN: 0023-432X), Vol.52, 5, 269-277.

5. Kukla D., Grzywna P., Karczewski R., Evaluation of the development of fatigue damage based on changes in strain and eddy current parameters in subsequent cycles load [in Polish], Welding Technology Review, 5 (2014), 23-31,

6. Khan S.H., Farhad A., usair Khan A., Iqbal M.A., Eddy current detection of changes in stainless steel after cold reduction, Computational Materials Science, Vol. 43 (2008), 623-628.

7. Kashefi M., Kahrobaee S., Nateq M.H., On the Relationship of Magnetic Response to Microstructure in Cast Iron and Steel Parts, Journal of Materials Engineering and Performance, 21 (2012), 1520-1525. 